\title{
A PROBLEM IN GEOMETRIC PROBABILITY
}

\author{
J. G. WENDEL ${ }^{1}$
}

Let $N$ points be scattered at random on the surface of the unit sphere in $n$-space. The problem of the title is to evaluate $p_{n, N}$, the probability that all the points lie on some hemisphere. I shall show that

$$
p_{n, N}=2^{-N+1} \sum_{k=0}^{n-1}\left(\begin{array}{c}
N-1 \\
k
\end{array}\right) \text {. }
$$

I first heard of the problem from L. J. Savage, who had been challenged by R. E. Machol to evaluate $p_{3,4}$. Savage showed that $p_{3,4}=\frac{7}{8}$, and more generally that

$$
p_{n, n+1}=1-2^{-n} .
$$

Then I was able to obtain the relation

$$
p_{n, n+2}=1-(n+2) 2^{-(n+1)} .
$$

and D. A. Darling proved that $p_{2, N}=N \cdot 2^{-N+1}$, which on setting $N=n+2$ became

$$
p_{2, n+2}=(n+2) 2^{-(n+1)} .
$$

Equations (3) and (4) suggested the attractive "duality relation"

$$
p_{m, m+n}+p_{n, m+n}=1 \text {, }
$$

which was found to hold generally. The results (2), (3) and (5) then led to the conjecture (1). Since (5) is a corollary to (1) it seems superflous to give a separate proof; instead I proceed now to the proof of (1), and in a slightly more general setting.

Let $x_{1}, x_{2}, \ldots, x_{N}$ be random vectors in $E^{n}$ whose joint distribution is invariant under all reflections through the origin and is such that with probability one all subsets of size $n$ are linearly independent; for example. the $x_{j}$ may be uniformly and independently distributed over the surface of the unit sphere. The probability $p_{n, N}$ is now interpreted as the probability that all $x_{j}$ lie in a half-space. i.e. that for some vector $y$ the inner

Received August 29, 1962.

1 Supported by U.S. National Science Foundation Contract G 19117. 
products $\left(y, x_{j}\right)$ are all positive. I shall show that $p_{n, .}$ satisfies the recurrence relation

$$
p_{n, N}=\frac{1}{2}\left(p_{n, N-1}+p_{n-1, N-1}\right) .
$$

Since the right member of (1) also satisfies (6), together with the evident boundary conditions $p_{1, N}=2^{-N+1}, p_{n, N}=1$ if $N \leqq n$, this will complete the proof of (1).

Proof of (6). It is sufficient to evaluate the corresponding conditional probability when the $x_{j}$ are non-zero and lie on fixed lines through the origin. Suppose that $y$ is perpendicular to none of these lines. Then the sequence $s_{y}=\left\{\operatorname{sgn}\left(y, x_{j}\right)\right\}$ is a random point in the set $S=\{s\}$ of all ordered $N$-tuples consisting of plus and minus signs. A specified $s$ is said to occur if there is a $y$ such that $s_{y}=s$. Let $A_{s}$ be the event that $s$ occurs, and let $I_{s}$ be the indicator of $A_{s}$. By definition $p_{n, N}=\operatorname{Pr}\left\{A_{s_{0}}\right\}$. where $s_{0}=(+,+, \ldots,+)$. Since any $s$ can be changed into any other by reflecting appropriate $x_{j}$ through the origin it follows that all $A_{s}$ are equally likely. Hence

$$
2^{N} p_{n, N}=\sum_{s} \operatorname{Pr}\left\{A_{s}\right\}=E\left(\sum_{s} I_{s}\right)=E(Q),
$$

say, with $Q=Q_{n, N}=\Sigma_{s} I_{s}$ being the number of different $s$ that occur.

Ostensibly $Q$ is a random variable, but in fact a simple argument now shows that $Q$ is a constant not depending on the directions of the fixed lines, providing of course that they are linearly independent in sets of $n$. Let $X_{j}$ be the hyperplane perpendicular to $x_{j}$. Then $Q$ is just the number of components (maximal connected subsets) complementary to all the $X_{j}$ in $E^{n}$, because each component consists of all the vectors $y$ for which $s_{y}$ has a fixed value.

In order to count the components, consider the effect of deleting one hyperplane, say $X_{N}$. There remain $N-1$ hyperplanes, with complementary set composed of $Q_{n, N-1}$ components. These components are of two kinds: (i) those which meet $X_{N}$, and (ii) those not meeting $X_{N}$. In an obvious notation we have $Q_{n, N-1}=Q^{(i)}+Q^{(i i)}$. When $X_{N}$ is restored it cuts each component of type (i) into two and does not disturb the others. Therefore $Q_{n, N}=2 Q^{(i)}+Q^{(i i)}$. It follows that

$$
Q_{n, N}=Q_{n, N-1}+Q^{(i)} \text {. }
$$

I claim now that $Q^{(i)}=Q_{n-1, N-1}$. In fact, the sets $X_{j} \cap X_{N}$ are hyperplanes in the $(n-1)$-dimensional space $X_{N}$, and their normals are linearly independent in sets of $n-1$. Therefore $X_{N}-\bigcup_{j=1}^{N-1}\left(X_{j} \cap X_{N}\right)$ has $Q_{n-1, N-1}$ components in $X_{N}$, and it is easy to see that these are just the intersec- 
tions of the original type (i) components with $X_{N}$, establishing the claim. Substituting into (7) and recalling that $Q_{n, N}=2^{N} p_{n, N}$ we obtain (6). This completes the proof.

The argument given above is essentially the same as that presented by Schläfli [1, pp. 209-212], but is included here for the sake of completeness. I am obliged to H. S. M. Coxeter for the reference. It may also be remarked that the form of the result (1) shows that $p_{n, N}$ equals the probability that in tossing an honest coin repeatedly the $n$ 'th "head" occurs on or after the $N$ 'th toss. But it does not seem possible to find an isomorphism between coin-tossing and the given problem that would make the result immediate.

\section{REFERENCE}

1. Ludwig Schläfli, Gesammelte mathematische Abhandlungen I, Basel, 1950.

UNIVERSITY OF MICHIGAN, ANN ARBOR, MICH., U.S.A.

AND

UNIVERSITY OF AARHUS, DENMARK 
